\title{
A Multi-Point of View 3D Camera System for Minimally Invasive Surgery
}

\author{
M. Silvestri ${ }^{\mathrm{a}}$, T. Ranzani ${ }^{\mathrm{a}}$, A. Argiolas ${ }^{\mathrm{a}}$, M. Vatteroni ${ }^{\mathrm{a}}$, A. \\ Menciassi $^{\mathrm{a}}$
}

${ }^{a}$ The Biorobotic Institute, Scuola Superiore Sant'Anna, viale R. Piaggio, 34, 56025 Pontedera (PI), Italy

\begin{abstract}
As a result of the rapid spreading of stereoscopy in the consumer market, three-dimensional (3D) vision systems are replacing two-dimensional devices. A fast growing technology in the 3D visualization systems market is multi-views autostereoscopic displays (ADs). However, these devices have not found yet direct application in minimally invasive surgery (MIS), mainly because of the encumbrance of the acquisition system. Indeed, at least five aligned camera modules must be embedded in a device insertable through a laparoscopic trocar. The aim of this work is the development of a miniaturized acquisition system for MIS, which can be interfaced with multi-views ADs, thus paving the way to the use of such devices in surgery. The device dimensions are compatible with MIS access ports and a magnetic link allows the user to anchor and move the acquisition system inside the abdomen.

(C) 2012 The Authors. Published by Elsevier Ltd. Selection and/or peer-review under responsibility of the Symposium Cracoviense Sp. z.o.o.
\end{abstract}

Keywords: 3D Display, Autostereoscopic Display, Minimally Invasive Surgery.

\section{Introduction}

Despite the number of surgical procedures being performed using a minimally invasive approach is increasing, various technical challenges are still open. A hot topic is vision; indeed, the loss of a direct view of the operative field remains one of the most critical issues. Although standard laparoscopic imaging systems provide optimal performance in terms of image quality, details, and colour sharpness, the loss of depth perception, due to the two-dimensional (2D) visualization, is the main limitation. Progresses in imaging system technologies have made three-dimensional (3D) view of the surgical field feasible and appealing. Currently, only single-user platforms are available on the market, and they need dedicated accessories for 3D visualization [1, 2]. The image acquisition systems for these devices are rigid 
laparoscopes, that are limited both in terms of field of view of the surgical scenario and manoeuvrability. Moreover, conflicts between the endoscopist manoeuvring the camera, and the surgeon controlling the assistive and operative instruments, are possible [3]. To overcome these limitations, different devices have been presented in literature. Such devices can be inserted at the beginning of the intervention and fixed to the abdomen by suturing [4] or exploiting a magnetic link between internally embedded permanent magnets (IPM) and external permanent magnets (EPM) [5, 6]. In this way, the access trocar is not occupied by the vision system during the surgical procedure. Additionally, the use of magnetism can increase the manoeuvrability of the device allowing a rapid repositioning of the system inside the abdomen. In particular, Simi et al [6] explored the use of two-view autostereoscopic display (AD) in a magnetically held device, thus enabling 3D perception to the whole surgical staff and avoiding the need of additional accessories [7]. Although two-views ADs demonstrated their effectiveness in providing 3D cues of the surgical field [8], the current technology of multi-views ADs may further improve surgical performance by decreasing ocular fatigue and improving the 3D perception. Currently, multi-views ADs have not yet found a direct application in MIS, mainly because of the encumbrance of the acquisition system: at least five aligned camera modules must be embedded in a device that has to be inserted through the surgical incision. The aim of this work is the development of a miniaturized and magnetically actuated acquisition system for MIS, which can be interfaced to multi-views ADs by embedding up to seven cameras, thus paving the way to the use of such devices in surgery.

\section{Materials and methods}

One of the main issues, in designing an acquisition system for multi-views ADs, was the embedding of at least five aligned points of view in a device with critical dimension requirements. A simple solution was found by the authors in the implementation of a module which can be inserted in the longitudinal direction, while anchored and manoeuvred in the horizontal one. The presented device aligns the cameras within an internal unit, keeping constrained the overall dimensions and enabling the easy and quick positioning of the device on the abdominal wall. The device is based on an internal unit, for hosting and aligning properly up to 7 points of view, magnetically linked to an external handle for anchoring, positioning and tilting the vision acquisition system along the abdomen (Fig. 1).

(a)

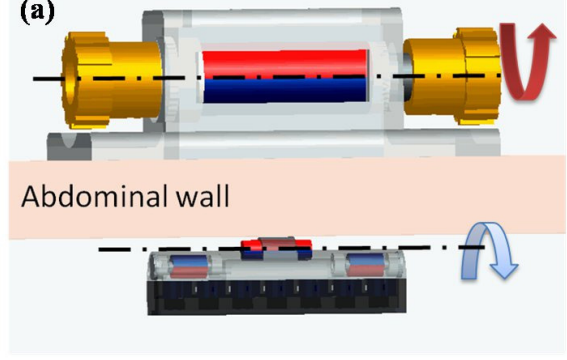

(b)

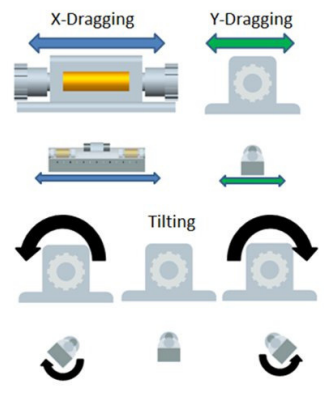

Fig. 1. (a) CAD-view of complete device. b) Scheme of the magnetically transmitted degrees of freedom.

The video acquisition system is based on a CMOS STMicroelectronics camera module, $1616 \times 1208$ in resolution and $5 \mathrm{~mm} \times 5 \mathrm{~mm} \times 3.8 \mathrm{~mm}$ in size (Fig. 2(a)). The imager is embedded on a Printed Circuit Board $(\mathrm{PCB})$ of $10 \mathrm{~mm}$ in diameter, in order to meet the requirements that are declared by $\mathrm{AD}$ manufacturers [9]. The PCB embeds also four white high efficiently Light Emitting Diodes (LEDs) for the illumination system. The LEDs are arranged radially around the camera in order to keep the modularity of the device. Data transmission and power supply to a single camera module are ensured by a 
wired connection with an acquisition board (Fig. 2(b)) which also allows visualizing and storing the image stream on a laptop. From the mechanical point of view, the insertable device is designed for both providing correct position and orientation of the whole vision acquisition system, and hosting three cylindrical IPMs for anchoring and moving the device. An external handle was designed for hosting the EPM. The EPM can be manually rotated along its axis for providing the tilting of the inner device and guaranteeing the axial force necessary for the anchoring to the abdominal wall. FEM simulations were performed using COMSOL Multiphysics ${ }^{\mathrm{TM}}$ for dimensioning the magnets embedded in the external unit and in the internal one. The maintenance of the magnetic anchoring at the various tilting angles and the effectiveness of the tilt degree of freedom (DOF) were considered as the design requirements.
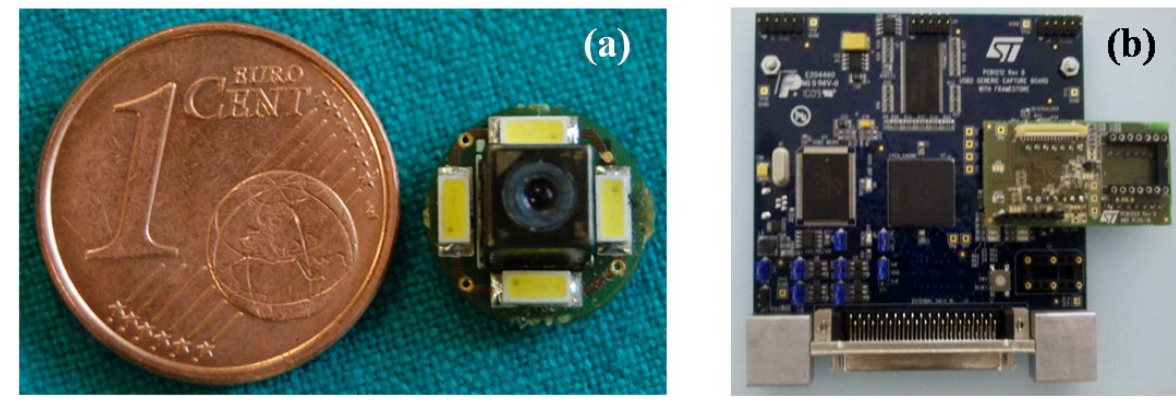

Fig. 2. (a) The designed PCB of the single acquisition module, embedding the camera and the illumination system; (b) The acquisition system board.

\section{Results}

In order to test the overall device functionality, the acquisition system along with the mechanical functionalities were verified. One of the main constraints in designing a multi-views acquisition system was to guarantee the correct alignment of the cameras in order to avoid artifact or ocular fatigue during the visualization. Therefore, the device was fixed on a support and the images acquired by five cameras were stored (Fig. 3) and elaborated using Matlab ${ }^{\mathrm{TM}}$. The resulting alignment error was $\pm 0.24^{\circ}$, which is less than the physiological limit $\left( \pm 0.32^{\circ}\right)$, thus not introducing an uncomfortable vertical distortion [10].
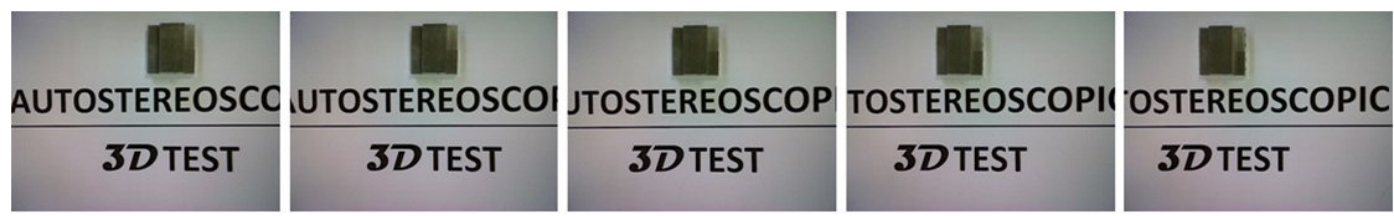

Fig. 3. Images acquired for testing the alignment of the PCBs inside the internal unit.
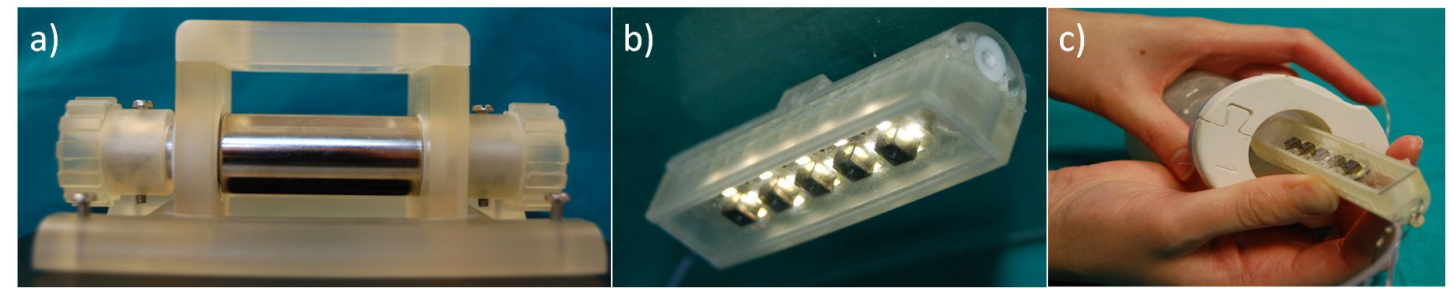

Fig. 4. (a) Insertable unit. (b) External handle; (c) Insertion of the acquisition system in a trocar for minimally invasive surgery. 
The insertable unit and the external handle were designed and fabricated by rapid prototyping (Fig. 4 a, b). The insertable device fits in a boundary box of $\mathrm{L} \times \mathrm{W} \times \mathrm{H}=73 \mathrm{~mm} \times 17 \mathrm{~mm} \times 21 \mathrm{~mm}$, thus allowing it to be introduced from the incision performed during a MIS procedure (Fig. $4 \mathrm{c}$ ). The dimensions of the external handle are $\mathrm{L} \times \mathrm{W} \times \mathrm{H}=120 \mathrm{~mm} \times 70 \mathrm{~mm} \times 43 \mathrm{~mm}$. Assuming a $3 \mathrm{~cm}$ thickness of the abdominal wall, the computed magnetic force for anchoring the internal unit (up to $1.75 \mathrm{~N}$ ) is sufficient to support the frame weight, including the cameras and illumination modules (about $0.02 \mathrm{Kg}$ ). The induced torque (up to $0.02 \mathrm{Nm}$ ) allows a tilting of the internal unit of $\pm 60^{\circ}$, while keeping a sufficient anchoring magnetic force for holding the device (a safety factor of 6 is guaranteed). Simulation results show that the maximum allowable tilting angle before the lost of the magnetic link is around $100^{\circ}$, that anyhow cannot be realistically reached because of the contact between the internal frame and the abdominal wall.

\section{Conclusions}

Although multi-views ADs present a lot of potential advantages in the MIS scenario they still do not have a direct surgical application, mainly because of technical limitations. Indeed, it is difficult to integrate more than 5 points of view in a miniaturized device insertable through a trocar. The presented system overcomes these problems by the integration of up to 7 camera modules inside a frame, matching laparoscopic trocar dimensions. The flexibility of the presented device allows the interface with a wide range of ADs. Additionally, the magnetic coupling guaranties the simple positioning and manoeuvrability into the patient's body. Future work is related to the development of a custom interface which will enable the complete video stream acquisition to be visualized by a multi-views $\mathrm{AD}$.

\section{Acknowledgements}

This work was supported in part by the European Commission within the framework of the ARAKNES European Project EU/IST-2008-224565. Authors would like to thank STMicroelectronics and Nichia for the components used in this work.

\section{References}

[1] J. H. Kaouk et al, Robotic single-port transumbilical surgery in humans: initial report, BJU international, 103 (2008) 366 369.

[2] KarlStorz website: http://www.karlstorz.com/.

[3] P. Weibl, Current Limitations and Perspectives in Single Port Surgery: Pros and Cons Laparo-Endoscopic Single-Site Surgery (LESS) for Renal Surgery, Diagnostic and Therapeutic Endoscopy, 2010 (2010), ID 759431.

[4] T. Hu et al, Insertable Stereoscopic 3D Surgical Imaging Device with Pan and Tilt, Proceedings of the 2nd Biennial IEEE/RAS-EMBS, Scottsdale, AZ, USA, October 19-22, 2008.

[5] J. Cadeddu et al, Novel magnetically guided intra-abdominal camera to facilitate laparoendoscopic single-site surgery: initial human experience, Surgical Endoscopy, 23 (2009) 1894-1899.

[6] M. Simi et al, Magnetically Activated StereoscopicVision System for Laparoendoscopic Single Site Surgery, IEEE Trans. Mechatronichs, in press.

[7] H. Urey et al, State of the Art in Stereoscopic and Autostereoscopic Displays, Proc IEEE, 99 (2011) 540-555.

[8] M. Silvestri et al, Autostereoscopic Three-Dimensional Viewer Evaluation Through Comparison with Conventional Interfaces in Laparoscopic Surgery, Surgical Innovation 18 (2011) 223-230.

[9] Tridelity website: http://www.tridelity.com/.

[10] Stereoscopic video Dataset; Image and Video Systems Lab; Korea Advanced Institute of Science and Technology (KAIST),Daejeon, Korea. 